\title{
NSW HEALTH CLINICAL INFORMATION ACCESS PROGRAM
}

Dianne Ayres and Michelle Wensley

Clinical Systems

NSW Department of Health

\section{HOWTHE PROGRAM EVOLVED}

In 1996 the NSW Health Clinical Systems Steering Committee, which governs a number of clinical committees, endorsed the development of a Web site to provide information to clinicians in the NSW public health system. As a result, the Clinical Information Access Project (CIAP) Web site was established on 4th July, 1997. The CIAP provides access to a comprehensive range of peerreviewed information 24 hours a day, seven days a week, via the Internet and Intranet. Utilisation of the Web site has steadily increased, peaking at 840,000 'hits' in the month of March 2000, which is an all-time monthly record. The site has averaged 340,000 'hits' per month since July 1999, and has recorded over eight million 'hits' since its inception in 1997.

\section{THE OBJECTIVES OF CIAP}

The primary objectives of making this information available on the Web were to:

- provide support for decision making within the public health system

- promote evidence-based practice

- improve communications at the point of care (that is, wherever a patient is located).
Clinicians were therefore consulted to ascertain the information resources needed to meet these objectives. A CIAP Committee was convened to oversee the project, including the acquisition and approval of information resources. The pre-implementation survey, completed in September 1997, confirmed the top 12 priorities of NSW clinicians for information resources on the Web (Figure $6)$.

\section{THE CONTENT OF CIAP}

Content has grown over the past two years in response to requests by clinicians and the following on-line information is now available via the CIAP Web site:

- Medline and CINAHL with over 12 million citations and links to 30 full text medical and nursing journals

- the full Cochrane Library with over 700 evidencebased reviews

- PsycINFO: psychiatry and psychology databases linked to 10 full text mental health journals

- MIMS for prescription decision-support

- Consumer Medicines Information (CMIs) pamphlets linked to MIMS

- HealthStar for health services, technology, research and quality management literature

- Antibiotic Guidelines (10th edition)

- Micromedex: Poisindex, Toxpoints, Emergindex, DrugREAX and Martindale databases

\section{FIGURE 6}

THE TOP 12 PRIORITIES OF NSW CLINICIANS FOR INFORMATION RESOURCES ON THE WEB

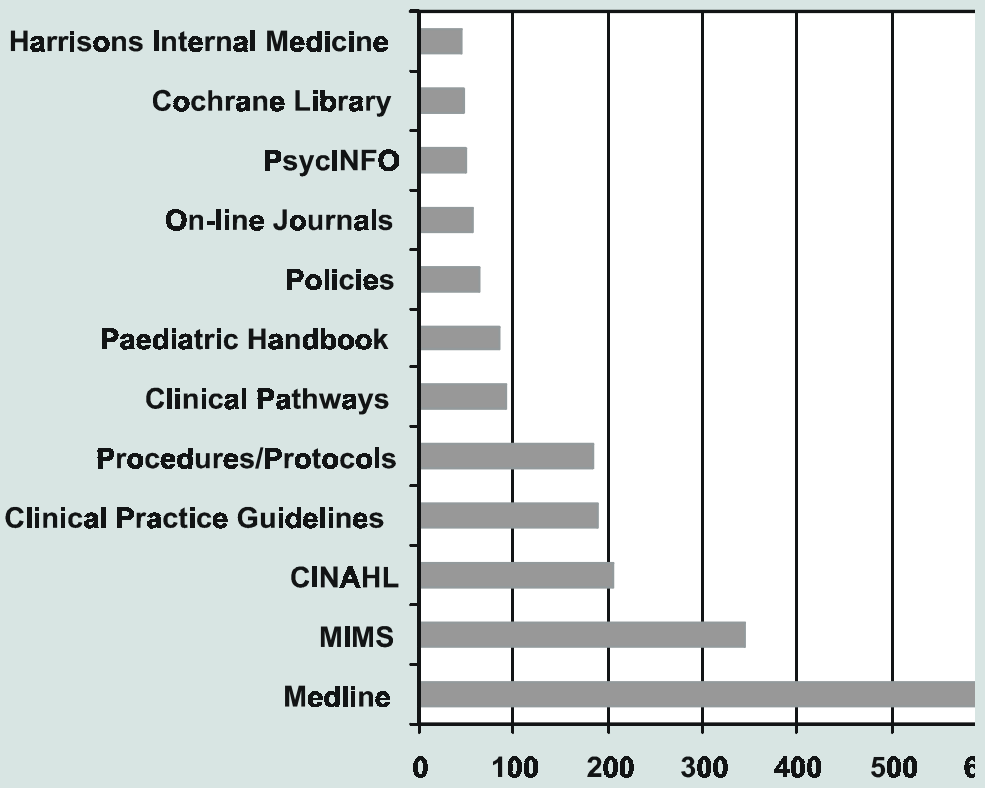


- Harrison's Textbook of Internal Medicine (14th edition)

- Primary Care Online: nine textbooks for medicine, nursing, drugs and diagnostic tests

- Interactive ECG: a tutorial of ECG case studies and tests

- Internet and email tutorials

- policies, procedures, protocols, clinical practice guidelines and clinical pathways from contributing Area Health Services in NSW

- NSW Health policies and guidelines

- list servers to improve communications between clinicians

- Therapeutic Guidelines: Analgesic, Gastrointestinal, Neurology, Endocrinology and Psychotropic Guidelines (on trial Feb-May 2000)

- links to International and National clinical Web sites

- Public Health Web sites including those of the Public Health Division, NSW Department of Health; the NSW Public Health Bulletin; the Public Health Association of Australia (PHA); and the Public Health Division, Health and Community Services, Victoria.

Other relevant Web sites can be added to this list by advising CIAP via the Feedback link on the CIAP Web site.

\section{ACCESS}

While the CIAP knowledge databases (MIMS, Cochrane, etc.) are protected by password, they are accessible to any health professional employed in the NSW public health system. Passwords are available by contacting the local Area Health Service CIAP representative at www.clininfo.health.nsw.gov.au/contacts/index.html

\section{CIAP ON-LINE SURVEY}

Feedback from clinicians has been positive and there is a continuous high demand for education on how to effectively search the databases. In 1998, the CIAP received:

- the Data Management Association (DAMA) Australia Achievement Award for Excellence in Information Management;

- the Australian Library and Information Association (ALIA) NSW Branch Merit Award for Services to Rural and Remote Users and the Community;

and was nominated in the top 10 medical Web sites in Australia by $P C$ Authority.

In 2000, CIAP will continue to provide on-line access to information that supports evidence based health care in NSW. F $^{2}$

For further information please contact Michelle Wensley, Senior Project Officer, Clinical Systems, NSW Department of Health; telephone (02) 9391 9742; or email mwens@doh.health.nsw.gov.au. The CIAP Web site can be found at:

www.clininfo.health.nsw.gov.au.

\section{ERRATUM}

In the March 2000 issue of the NSW Public Health Bulletin (Volume 11, Number 3, page 31), Figure 2 in the article by Scanlon and Raphael, 'Building capacity for promotion, prevention and early intervention in mental health', contained a small error. The left arrow of the heading Early Intervention should have stopped at the section of the pie chart labelled Indicated (under Prevention). A corrected figure is printed below.

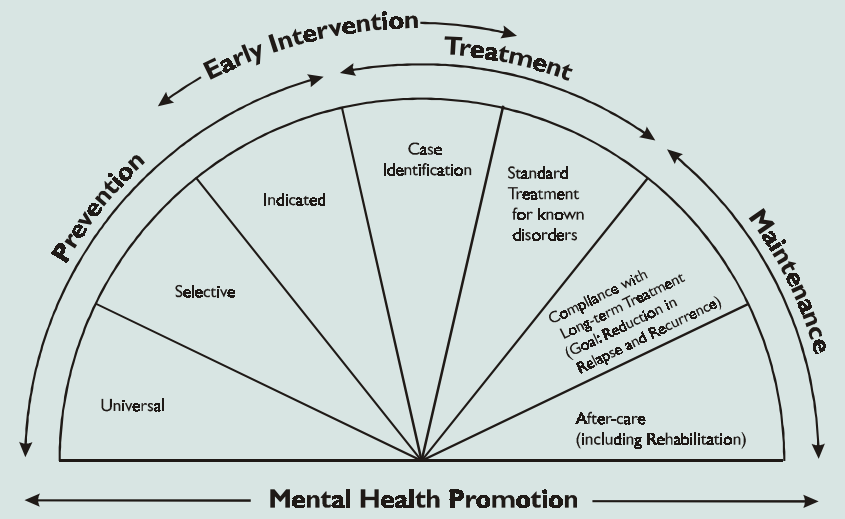

